POS $\quad$ PROCEEDINGS

\title{
Fast Calorimeter Simulation in LHCb
}

\author{
Fedor Ratnikov*广 \\ NRU Higher School of Economics, Moscow, Russia \\ E-mail: Fedor.Ratnikov@cern.ch
}

\section{Egor Zakharov}

Skolkovo Institute of Science and Technology, Moscow, Russia

In HEP experiments, CPU resources required by MC simulations constantly grow and become a very large fraction of the required total computing power (greater than $75 \%$ ). At the same time, the pace of performance improvements from technology is slowing down. The only solution is a more efficient use of resources. LHC experiments seek options for simulating higher statistics events in a faster way. A key to the success of this strategy is the possibility of enabling fast simulation options in a common framework with minimal action by the final user. In this paper, we describe the solution to selectively exclude particles from being simulated by the Geant 4 toolkit and to insert the corresponding hits generated in a faster way. The approach, integrated within the Geant4 toolkit, has been applied to the LHCb calorimeter, but it could also be used for other subdetectors. The hits generation can be carried out by any external tool, such as a static library of showers or more complex machine-learning techniques. Generative models, which are nowadays widely used for computer vision and image processing, are being studied as a candidate to accelerate the generation of showers in the calorimeter. We present how both approaches can be applied to the LHCb calorimeter simulation, their advantages as well as their drawbacks.

The 39th International Conference on High Energy Physics (ICHEP2018)

4-11 July, 2018

Seoul, Korea

\footnotetext{
* Speaker.

${ }^{\dagger}$ on behalf of the LHCb collaboration
} 


\section{Introduction}

During Run 2, the simulation of physics events at LHCb took about $80 \%$ of the distributed computing resources, which were available to the experiment [1]. The increase in the number of events, that will need to be simulated in Run 3 to match the higher luminosity and trigger rate, will place an extreme burden on the computing resources. To face this situation, it is necessary to develop new ways to significantly increase the speed of the simulation.

In a typical minimum bias event, calorimeter system takes about 55\% of the CPU time used by Geant4 [2] to simulate particle transportation. Given this number, in the effort of developing a faster detector simulation, it is natural to start from the calorimeter.

A number of fast simulation options are available or are under development in LHCb to complement the standard Geant 4 based simulation. In this short paper, we consider two approaches to speed up a simulation of response in the electromagnetic calorimeter (ECAL): using pre-simulated library of calorimeter responses, and generative model trained on the pre-simulated sample.

The LHCb detector [3] is equipped with ECAL, that employs a "shashlik" technology: alternating $4 \mathrm{~mm}$ thick scintillator tiles and $2 \mathrm{~mm}$ thick lead plates are arranged perpendicular to the beam pipe. The detector is not longitudinally segmented but adopts a variable lateral segmentation, because the hit density varies by two orders of magnitude over the calorimeter surface. A segmentation into three different sections has been chosen for the ECAL: square cell sizes are of approximately 40, 60 and $120 \mathrm{~mm}$ in the inner, middle and outer regions, respectively.

\section{Library Approach}

Details for this approach may be found elsewhere [4]. To reduce the number of parameters in the produced response library, the cell transverse area is divided into small subregions, or "points". A library of points is built from simulated incident particles with fixed values for particle position and azimuthal angle. Hence, for a given particle species, only the binnings in energy and incidence angle remain. An example of point distribution in the ECAL, produced by an incident photon with energy $\mathrm{O}(1) \mathrm{GeV}$, is shown in Fig. 1a, top-left plot: each square of the grid represents the transverse area of an ECAL cell, and the colour scale indicates the deposited energy. First, two or more points, belonging to the same cell area and with associated energy below a given threshold, may be merged locally to simplify the collection by reducing the number of points stored in the library. This is exemplified in the top-right plot. Next, the points are shifted and rotated according to the position of incidence and azimuthal angle of the particle with respect to the values for which the library was built. This is a key aspect of the point library. The collection of points represents a good model of the shower projection in the transverse plane. Shift and rotation of this collection give a good description of the shower produced by a translated and rotated incident particle. The rotation of the points around the position of incidence of the particle is exemplified in the bottomleft plot. Finally, the calorimeter hits are created by summing the energies of those transformed points which fall into the same cell area. This is shown in the bottom-right plot, where the colour in the central region of the cell indicates the total deposited energy.

The accuracy of the calorimeter simulation, based on point libraries, has been tested through the comparison with the detailed simulation using photons, generated at the calorimeter entrance 

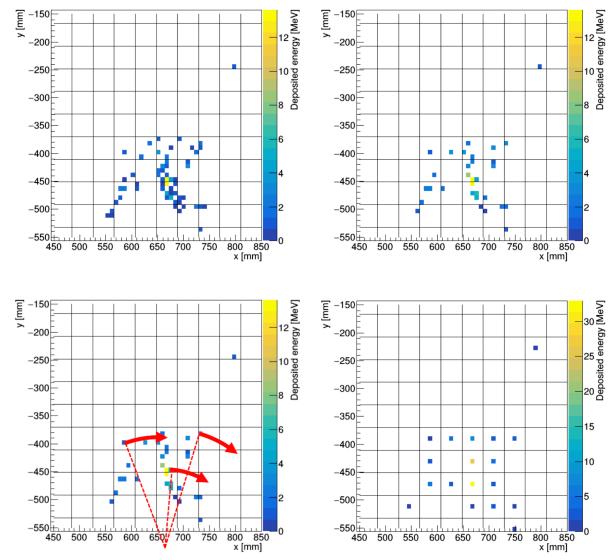

(a) Schematic illustration of the point library operating principle. More details are given in section 2 .
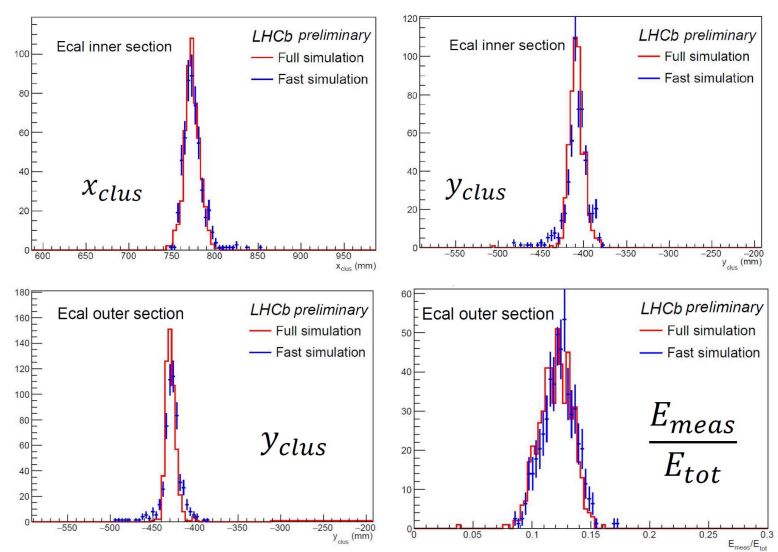

(b) Comparison of photons simulated with Geant4 ("Full simulation") and with the point libraries ("Fast simulation")

Figure 1: Library approach.

with various energies and angles of incidence. The coordinate of the hit cluster center is calculated as a weighted average of coordinates of the cluster cells. Results are presented in Fig. 1b. The top plots compare the cluster position distributions in the simpler case, where the entry point of the fully simulated photons coincides with the entry point used to build a response in the library. Therefore, rotation alone is enough for the procedure described above. The bottom plots correspond to a more complicated case, which compares distributions for cluster position and measured energy, where the fully simulated photons are generated in the outer sector. Therefore, the points had to be both rotated and shifted. However, the agreement does not worsen, that confirms the idea behind the point library.

\section{Generative Model Approach}

The idea of generic generative model approach is to treat simulations as a black-box, and to replace the traditional Monte Carlo simulation with a method based on Generative Adversarial Networks [5].

We use Wasserstein GAN [6] with gradient penalty, which is considered to be a state-of-the-art technique for the image production. The architecture of this Neural Network and details of training the generative model are presented elsewhere [7].

After the generative model is built and trained, we first compare the original clusters produced by full Geant 4 simulation with the clusters generated by the trained model for the same parameters of the incident particles: the same energy, the same direction, and the same position on the calorimeter face. Corresponding images for the four arbitrary parameter sets are presented in Fig. 2. These images demonstrate very good visual similarity between simulated and generated clusters.

Then, we perform the quantitative evaluation of the proposed simulation method. While generic evaluation methods for generative models exist, we base our evaluation on physics-driven 

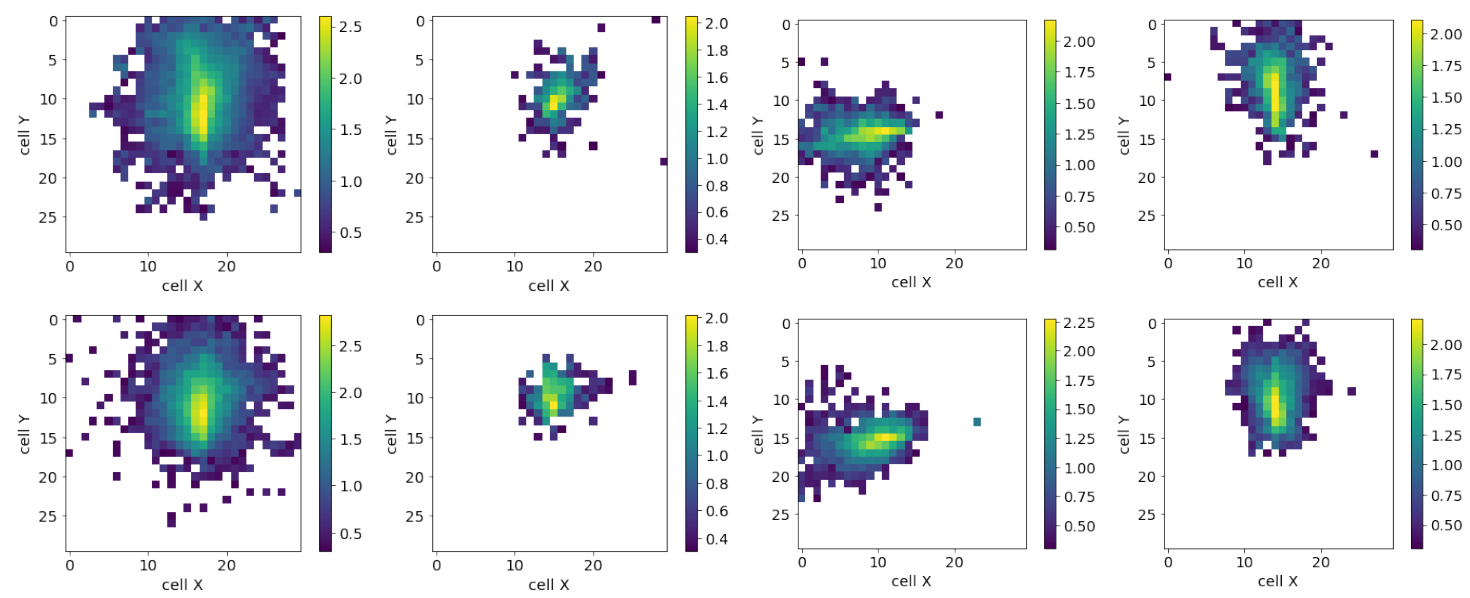

Figure 2: Showers generated with GEANT4 (first row) and the showers, simulated with our model (second row) for three different sets of input parameters. Color represents $\log _{10}\left(\frac{E}{\mathrm{MeV}}\right)$ for every cell.

similarity metrics. For this presentation, we selected only a few cluster properties, which essentially drive the cluster properties used in the reconstruction of the calorimeter objects and following physics analysis. If the initial particle direction is not perpendicular to the calorimeter face, the produced cluster is elongated in that direction. Therefore, we separately consider cluster widths in the direction of the initial particle and in the transverse direction. Spatial resolution, which is the distance between the center of mass of the cluster and the initial track projection to the shower max depth, is another important characteristic affecting physics properties of the cluster. Cluster sparsity, which is the fraction of cells with energies above some threshold, reflects marginal low energy properties of the generated clusters. These characteristics are presented in Fig. 3.

\section{Conclusions}

$\mathrm{LHCb}$ faces the current and future limitations of CPU resources with respect to the size of the necessary simulated samples. This limitation leads to an ongoing effort to develop fast simulation alternatives to the nominal detector simulation. In the detailed simulation based on Geant 4 , more than $50 \%$ of the CPU time is spent in the calorimeter system. We use different approaches to develop faster simulation of the calorimeter. Both library and generative model approaches are encouraging in terms of time gain and simulation accuracy. The use of a point library, as opposed to a more standard cell hit library, significantly improves the output accuracy for the same size of the library. We also confirm that Generative Adversarial Networks are a good candidate for fast simulation of high granularity detectors, typically considered for the next generation accelerators. We have successfully generated images of shower energy deposition with a condition on the particle parameters, such as the momentum and the coordinate, using modern generative deep neural network techniques. 


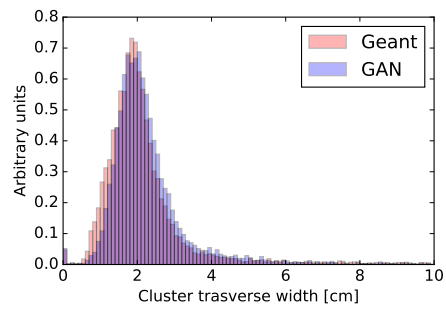

(a) The transverse width of real and generated clusters

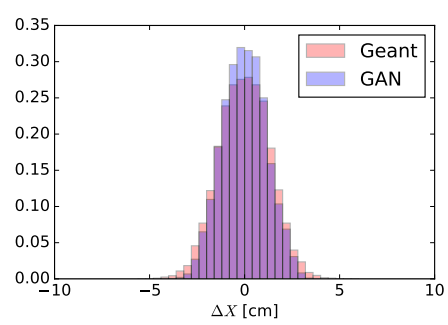

(c) $\Delta X$ between cluster center of mass and the true particle coordinate

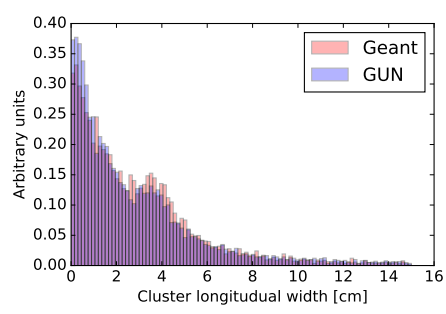

(b) The longitudinal width of real and generated clusters

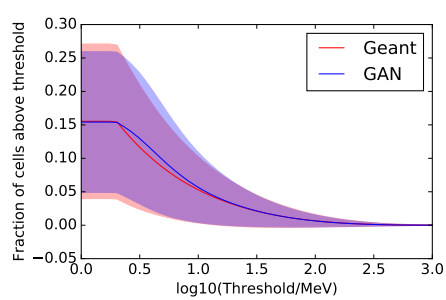

(d) The sparsity of real and generated clusters

Figure 3: Generated images quality evaluation including described physical characteristics.

\section{References}

[1] LHCb collaboration, LHCb Upgrade Software and Computing Technical Design Report, CERN-LHCC-2018-007, LHCb-TDR-017.

[2] S.Agostinelli et al.,(Geant4 Collaboration), Geant4: Asimulation toolkit,Nucl.Instrum. Methods Phys. Res., Sect. A 506, 250 (2003); J. Allison et al., (Geant4 Collaboration), IEEE Trans. Nucl. Sci. 53, 270 (2006).

[3] LHCb collaboration, A. A. Alves Jr. et al., The LHCb detector at the LHC, JINST 3 (2008) S08005.

[4] M.Rama and G.Vitali (LHCb collaboration), Calorimeter fast simulation based on hit libraries in the LHCb Gauss framework, proceedings of CHEP2018 conference, to be published in the EPJ Web of Conferences.

[5] I. Goodfellow, J. Pouget-Abadie, M. Mirza, B. Xu, D. Warde-Farley, S. Ozair, A. Courville, Y. Bengio, Generative adversarial nets, in Advances in neural information processing systems (2014), pp. 2672-2680.

[6] T.C. Wang, M.Y. Liu, J.Y. Zhu, G. Liu, A. Tao, J. Kautz, B. Catanzaro, arXiv preprint arXiv:1808.06601 (2018).

[7] V.Chekalina et al., Generative Models for Fast Calorimeter Simulation: LHCb case, proceedings of CHEP2018 conference, to be published in the EPJ Web of Conferences. 